\title{
Hamiltonian Dynamics and Adiabatic Invariants for Time-Dependent Superconducting Qubit-Oscillators and Resonators in Quantum Computing Systems
}

\author{
Jeong Ryeol Choi \\ Department of Radiologic Technology, Daegu Health College, Buk-gu, Daegu 702-722, Republic of Korea \\ Correspondence should be addressed to Jeong Ryeol Choi; choiardor@hanmail.net
}

Received 19 July 2015; Accepted 5 October 2015

Academic Editor: Boris G. Konopelchenko

Copyright (C) 2015 Jeong Ryeol Choi. This is an open access article distributed under the Creative Commons Attribution License, which permits unrestricted use, distribution, and reproduction in any medium, provided the original work is properly cited.

\begin{abstract}
An adiabatic invariant, which is a conserved quantity, is useful for studying quantum and classical properties of dynamical systems. Adiabatic invariants for time-dependent superconducting qubit-oscillator systems and resonators are investigated using the Liouville-von Neumann equation. At first, we derive an invariant for a simple superconducting qubit-oscillator through the introduction of its reduced Hamiltonian. Afterwards, an adiabatic invariant for a nanomechanical resonator linearly interfaced with a superconducting circuit, via a coupling with a time-dependent strength, is evaluated using the technique of unitary transformation. The accuracy of conservation for such invariant quantities is represented in detail. Based on the results of our developments in this paper, perturbation theory is applicable to the research of quantum characteristics of more complicated qubit systems that are described by a time-dependent Hamiltonian involving nonlinear terms.
\end{abstract}

\section{Introduction}

Interactions of light wave with matter are ubiquitous and have many applications in modern science and technology. An interesting research topic in this line is cavity quantum electrodynamics (cavity QED) which is relevant to the interaction of an atom with light waves inside a cavity. Meanwhile, it has been shown that the role of the cavity can be played by a superconducting device or others such as a nanomechanical oscillator or a transmission line [1-3]. Superconducting devices exhibit quantum interference and the electrons in them have the ability to act like qubits that are necessary in quantum computing systems. We mainly concentrate on these devices in this research. It is demonstrated from corresponding experiments that entanglement and the Rabi oscillations appear in a strongly coupled atom-cavity system as a result of quantum coherence between light and matter [3]. These features of spatial correlations enable us to do specific tasks regarding quantum information techniques such as quantum computing [4].

Recently, the study of superconducting qubit-oscillators in quantum computing systems with time-dependent parameters attracted considerable interest in the community of physics and information science [5-9]. In general, a high ability of computation with a tremendous speed can be achieved for particular mathematical problems from quantum computation on the basis of new paradigms known as superposition and entanglement. This is the most distinguishing feature of quantum computers, which cannot be mimicked by classical ones. The realization of producing universal quantum gates that fulfill quantum computations may require the use of geometric phase shifts of qubit systems along with the changes of suitable loops in the Hamiltonian under efficient quantum control. This can be performed through the use of superconducting nanocircuits [10].

Meanwhile, obtaining conserved quantities, that is, adiabatic invariants, is of importance in the research of the dynamics of quantum computing systems, because not only are they helpful for deepening the understanding of the system but also they can be used for studying the quantum and classical properties of the system itself. If the time derivative of a quantity results in zero, this quantity is a kind of exact invariant. On the other hand, an adiabatic invariant is a quantity in which its time derivative gives 
nearly zero under a slow variation of the Hamiltonian with time. The first proposal of adiabatic invariants was sprung by a pioneering work of Burgers [11], which is for a particular class of Hamiltonian systems. Soon after, many other researches concerning adiabatic invariants were carried out via the introduction of the Hamiltonian of a given system [12-18]. Adiabatic invariants for a linear harmonic oscillator have been studied by Dykhne in connection with quantum transitions [15]. Quantum algorithms for adiabatic quantum computation, which are robust to decoherence and not affected by certain noises, were developed [19]. A hybrid quantum simulation model composed of adiabatically controlled simulation registers connected to a qubit was proposed as well [20].

We will find adiabatic invariants for the Hamiltonians that describe the mechanics of qubit-oscillators and resonators in quantum computing systems that are characterized by time-varying parameters. Concerning this, the identification of adiabatic invariants for a time-dependent Hamiltonian system with complicated time-variable parameters may be a significant challenge. We will first address the invariant of a simple superconducting qubit-oscillator. Afterwards, an adiabatic invariant for a nanomechanical resonator coupled to a superconducting resonator, which is described by a complicated Hamiltonian involving a cross term, will be investigated. The Liouville-von Neumann equation will be used in order to derive conservative quantities for such dynamical systems.

\section{A Simple Superconducting Qubit-Oscillator}

Let us consider a simple superconducting qubit-oscillator of which the effective reduced Hamiltonian is given by Equation (2.2) of [21]. Typically, the two lowest energy levels of quantized states in the anharmonic LC resonator are regarded as a set of a qubit. In this case, the system is described by the Hamiltonian of the form [21]

$$
\widehat{H}_{A}=\hbar \omega\left(\widehat{a}^{\dagger} \widehat{a}+\frac{1}{2}\right)+\sqrt{\hbar} \delta(t)\left(\widehat{a}+\widehat{a}^{\dagger}\right),
$$

where $\delta(t)$ is the time-dependent coupling strength. Here, $\widehat{a}$ is the annihilation operator of the simple harmonic oscillator:

$$
\widehat{a}=\sqrt{\frac{L \omega}{2 \hbar}} \widehat{q}+\frac{i \widehat{p}}{\sqrt{2 \hbar L \omega}},
$$

with $\hat{p}=-i \hbar \partial / \partial q$, where $L$ is the inductance of the LC resonator. According to the usual convention, $\hat{a}^{\dagger}$ is Hermitian adjoint of $\widehat{a}$, and this rule will also be applied to other ladder operators that will be introduced later. In many cases of previous researches, the factor $1 / 2$ given in (1) is neglected for simplicity. Using (2) and its Hermitian adjoint, we can rewrite the Hamiltonian to be

$$
\widehat{H}_{A}=\frac{\widehat{p}^{2}}{2 L}+\frac{1}{2} L \omega^{2} \widehat{q}^{2}+\sqrt{2 L \omega} \delta(t) \hat{q} .
$$

The classical equation of motion for this system is given by

$$
\frac{d^{2} q}{d t^{2}}+\omega^{2} q=-\sqrt{\frac{2 \omega}{L}} \delta(t) .
$$

Although an (adiabatic) invariant is obtained under the assumption that its total change during a physical process with time-variable parameters is zero, it is represented in terms of $t$. A quantum (adiabatic) invariant is obtained from the following Liouville-von Neumann equation:

$$
\frac{d \widehat{I}_{A}}{d t}=\frac{\partial \widehat{I}_{A}}{\partial t}+\frac{\left[\widehat{I}_{A}, \widehat{H}_{A}\right]}{i \hbar}=0 .
$$

From a straightforward evaluation with this equation using (3), we have

$$
\widehat{I}_{A}=\frac{\left[\widehat{p}-P_{p}(t)\right]^{2}}{2 L}+\frac{1}{2} L \omega^{2}\left[\widehat{q}-Q_{p}(t)\right]^{2},
$$

where $Q_{p}(t)$ is a particular solution of $(4)$ and $P_{p}(t)=$ $L d Q_{p}(t) / d t$. Most previous research findings for adiabatic invariants, such as the research based on the method of Noether's theory [22], were performed under the supposition of the slow change of time-variables; however, the derivation of the invariant given in (6) does not require such a condition as you can see. This invariant in fact does not vary with time. It is an exact constant of motion for the given system, even for the case that the parameter $\delta(t)$ undergoes fast change with time.

\section{A Nanoresonator Coupled to a Superconducting Resonator}

The theorem of adiabatic invariants originally developed for slowly time-varying systems in one-dimension may also be extended to more complicated cases. Now let us consider a nanomechanical resonator interfaced with a superconducting circuit in a linear way. In this case, the Hamiltonian is represented as (see Equation (2) of [23] and Equation (31) of [24])

$$
\begin{aligned}
\widehat{H}_{B}= & \hbar \omega\left(\hat{a}_{1}^{\dagger} \widehat{a}_{1}+\frac{1}{2}\right)+\hbar \Omega\left(\hat{a}_{2}^{\dagger} \widehat{a}_{2}+\frac{1}{2}\right) \\
& +\hbar \lambda(t)\left(\widehat{a}_{1}+\hat{a}_{1}^{\dagger}\right)\left(\widehat{a}_{2}+\hat{a}_{2}^{\dagger}\right),
\end{aligned}
$$

where $\widehat{a}_{1}$ and $\widehat{a}_{2}$ are annihilation operators for the nanoresonator and stripline, respectively, and $\lambda(t)$ is the timedependent coupling strength between the two oscillators. We can represent the two annihilation operators to be

$$
\begin{aligned}
& \widehat{a}_{1}=\sqrt{\frac{L_{n} \omega}{2 \hbar}} \widehat{q}_{1}+\frac{i \widehat{p}_{1}}{\sqrt{2 \hbar L_{n} \omega}}, \\
& \widehat{a}_{2}=\sqrt{\frac{L_{s} \Omega}{2 \hbar}} \widehat{q}_{2}+\frac{i \widehat{p}_{2}}{\sqrt{2 \hbar L_{s} \Omega}},
\end{aligned}
$$

with $\widehat{p}_{j}=-i \hbar \partial / \partial q_{j}$, where $L_{n}$ and $L_{s}$ are inductances for the nanomechanical resonator and the superconducting circuit, respectively. In terms of canonical variables, we can rewrite the Hamiltonian as

$$
\begin{aligned}
\widehat{H}_{B}= & \frac{\widehat{p}_{1}^{2}}{2 L_{n}}+\frac{\widehat{p}_{2}^{2}}{2 L_{s}}+\frac{1}{2} L_{n} \omega^{2} \widehat{q}_{1}^{2}+\frac{1}{2} L_{s} \Omega^{2} \widehat{q}_{2}^{2} \\
& +2 \lambda(t) \sqrt{L_{n} L_{s} \omega \Omega} \widehat{q}_{1} \widehat{q}_{2} .
\end{aligned}
$$


If we introduce two linearly independent real classical solutions, $\rho_{j, \mathrm{I}}(t)$ and $\rho_{j, \mathrm{II}}(t)$, of the following equations [14]

$$
\frac{d^{2} \rho_{j}}{d t^{2}}+\widetilde{\omega}_{j}^{2}(t) \rho_{j}=0,
$$

for each $j$, where $j=1,2$, and

$$
\begin{aligned}
\widetilde{\omega}_{1}^{2}(t)= & \omega^{2} \cos ^{2} \varphi(t)+\Omega^{2} \sin ^{2} \varphi(t) \\
& -4 \lambda(t) \sqrt{\omega \Omega} \cos \varphi(t) \sin \varphi(t), \\
\widetilde{\omega}_{2}^{2}(t)= & \omega^{2} \sin ^{2} \varphi(t)+\Omega^{2} \cos ^{2} \varphi(t) \\
& +4 \lambda(t) \sqrt{\omega \Omega} \cos \varphi(t) \sin \varphi(t), \\
\varphi(t)= & \frac{1}{2} \tan ^{-1}\left(\frac{4 \lambda(t) \sqrt{\omega \Omega}}{\Omega^{2}-\omega^{2}}\right),
\end{aligned}
$$

we can define the Wronskians in the form

$$
W_{j}=\rho_{j, \mathrm{I}}(t) \dot{\rho}_{j, \mathrm{II}}(t)-\dot{\rho}_{j, \mathrm{I}}(t) \rho_{j, \mathrm{II}}(t),
$$

which are time-constants. The quantities $W_{j}$ defined in this way are necessary for evaluating the adiabatic invariant of the system. Hence, they will be used in the development of our theory for the adiabatic invariant later.

Let us start from the Hamiltonian given in (9) in order to derive an adiabatic invariant of the system. This Hamiltonian is somewhat complicated because it is not only dependent on time, but also described in terms of a cross term $\widehat{q}_{1} \widehat{q}_{2}$. Hence, to simplify the problem, we will transform the Hamiltonian to a simple form using the unitary transformation method. To do so, let us introduce the following unitary operator:

$$
\begin{aligned}
\widehat{U}_{B}= & \exp \left[\frac{i}{\hbar}\left(\widehat{p}_{1} \widehat{q}_{1}+\widehat{q}_{1} \widehat{p}_{1}\right) \ln \left(\frac{L_{n}}{L_{s}}\right)^{1 / 8}\right] \\
\cdot & \exp \left[\frac{i}{\hbar}\left(\widehat{p}_{2} \widehat{q}_{2}+\widehat{q}_{2} \widehat{p}_{2}\right) \ln \left(\frac{L_{s}}{L_{n}}\right)^{1 / 8}\right] \\
& \cdot \exp \left[-\frac{i \varphi(t)}{\hbar}\left(\widehat{p}_{1} \widehat{q}_{2}-\widehat{p}_{2} \widehat{q}_{1}\right)\right] .
\end{aligned}
$$

Then, the Hamiltonian can be transformed using the relation

$$
\widehat{H}_{B}^{\prime}=\widehat{U}_{B}^{-1} \widehat{H}_{B} \widehat{U}_{B}-i \hbar \widehat{U}_{B}^{-1} \frac{\partial \widehat{U}_{B}}{\partial t} .
$$

Through a little evaluation using fundamental properties of quantum operators, we derive the transformed Hamiltonian in the form

$$
\begin{aligned}
\widehat{H}_{B}^{\prime}= & \frac{\widehat{p}_{1}^{2}+\widehat{p}_{2}^{2}}{2 \sqrt{L_{n} L_{s}}}+\frac{1}{2} \sqrt{L_{n} L} \widetilde{\omega}_{1}^{2}(t) \widehat{q}_{1}^{2}+\frac{1}{2} \sqrt{L_{n} L} \widetilde{\omega}_{2}^{2}(t) \widehat{q}_{2}^{2} \\
& +\beta(t) \dot{\lambda}(t)\left(\widehat{p}_{1} \widehat{q}_{2}-\widehat{p}_{2} \widehat{q}_{1}\right),
\end{aligned}
$$

where

$$
\beta(t)=-\frac{2 \sqrt{\omega \Omega}}{\Omega^{2}-\omega^{2}+16 \omega \Omega \lambda^{2}(t) /\left(\Omega^{2}-\omega^{2}\right)} .
$$

If we consider the adiabatic condition $\dot{\lambda}(t) \simeq 0$, this Hamiltonian reduces to

$$
\begin{aligned}
\widehat{H}_{B}^{\prime} \simeq & \frac{\widehat{p}_{1}^{2}+\widehat{p}_{2}^{2}}{2 \sqrt{L_{n} L_{s}}}+\frac{1}{2} \sqrt{L_{n} L_{s}} \widetilde{\omega}_{1}^{2}(t) \hat{q}_{1}^{2} \\
& +\frac{1}{2} \sqrt{L_{n} L_{s}} \widetilde{\omega}_{2}^{2}(t) \hat{q}_{2}^{2} .
\end{aligned}
$$

This decoupled Hamiltonian is much more simple than the original one given in (9). We will use this Hamiltonian in the subsequent development of our quantum theory, instead of (15), for convenience.

Similar to the previous case, the invariant operator in the transformed system should satisfy the relation

$$
\frac{d \widehat{I}_{B}^{\prime}}{d t}=\frac{\partial \widehat{I}_{B}^{\prime}}{\partial t}+\frac{\left[\widehat{I}_{B}^{\prime}, \widehat{H}_{B}^{\prime}\right]}{i \hbar}=0 .
$$

The direct evaluation of the invariant operator using this equation with (17) leads to

$$
\begin{aligned}
\widehat{I}_{B}^{\prime}= & \frac{\sqrt{L_{n} L_{s}}}{2} \\
& \cdot \sum_{j=1,2}\left[\frac{W_{j}^{2}}{\rho_{j}^{2}(t)} \hat{q}_{j}^{2}+\left(\dot{\rho}_{j}(t) \hat{q}_{j}-\frac{\rho_{j}(t)}{\sqrt{L_{n} L_{s}}} \widehat{p}_{j}\right)^{2}\right],
\end{aligned}
$$

where

$$
\rho_{j}(t)=\left[\rho_{j, \mathrm{I}}^{2}(t)+\rho_{j, \mathrm{II}}^{2}(t)\right]^{1 / 2} .
$$

From the inverse transformation of (19) using $\widehat{I}_{B}=\widehat{U}_{B} \widehat{I}_{B}^{\prime} \widehat{U}_{B}^{-1}$, we can obtain the adiabatic invariant in the original system as

$$
\begin{aligned}
\widehat{I}_{B} & =\frac{\sqrt{L_{n} L_{s}}}{2} \\
& \cdot \sum_{j=1,2}\left[\frac{W_{j}^{2}}{\rho_{j}^{2}(t)} \widehat{Q}_{j}^{2}+\left(\dot{\rho}_{j}(t) \widehat{Q}_{j}-\frac{\rho_{j}(t)}{\sqrt{L_{n} L_{s}}} \widehat{P}_{j}\right)^{2}\right],
\end{aligned}
$$

where

$$
\begin{aligned}
& \widehat{Q}_{1}=\left(\frac{L_{n}}{L_{s}}\right)^{1 / 4} \cos \varphi(t) \widehat{q}_{1}-\left(\frac{L_{s}}{L_{n}}\right)^{1 / 4} \sin \varphi(t) \widehat{q}_{2}, \\
& \widehat{Q}_{2}=\left(\frac{L_{n}}{L_{s}}\right)^{1 / 4} \sin \varphi(t) \widehat{q}_{1}+\left(\frac{L_{s}}{L_{n}}\right)^{1 / 4} \cos \varphi(t) \widehat{q}_{2}, \\
& \widehat{P}_{1}=\left(\frac{L_{s}}{L_{n}}\right)^{1 / 4} \cos \varphi(t) \widehat{p}_{1}-\left(\frac{L_{n}}{L_{s}}\right)^{1 / 4} \sin \varphi(t) \widehat{p}_{2}, \\
& \widehat{P}_{2}=\left(\frac{L_{s}}{L_{n}}\right)^{1 / 4} \sin \varphi(t) \widehat{p}_{1}+\left(\frac{L_{n}}{L_{s}}\right)^{1 / 4} \cos \varphi(t) \widehat{p}_{2} .
\end{aligned}
$$

Thus, the adiabatic invariant of the system is completely identified now. Because we have used the condition $\dot{\lambda}(t) \simeq 0$ in the derivation of the invariant quantity, (21) is valid only when $\lambda(t)$ varies sufficiently slowly. Hence, the accuracy of conservation for $\widehat{I}_{B}$ is determined by the degree of timevariance of $\lambda(t)$. Such accuracy for a conserved quantity is of theoretical interest in the general physics literature [16-18]. 


\section{Conclusion}

Adiabatic invariants for superconducting qubit-oscillator systems and nanomechanical resonators have been investigated. An exact invariant for a simple superconducting qubit-oscillator was evaluated. An adiabatic invariant for a nanoresonator coupled to a superconducting resonator was also obtained as shown in (21). The invariant $\widehat{I}_{B}$ for the latter case is more complicated than the former $\widehat{I}_{A}$. Notice that $\widehat{I}_{B}$ was derived under the assumption that the time-dependent parameter $\lambda(t)$ varies sufficiently slowly, while the derivation of $\widehat{I}_{A}$ required no such assumption. The concept of adiabatic invariants is important in both quantum and classical points of view $[25,26]$, because we can study the characteristics of the system by making use of them. In particular, these invariants enable us to derive Schrödinger solutions of the dynamical systems [13].

For a more complicated system, the evaluation of adiabatic invariants may be not an easy task. We do not know how to derive adiabatic invariants for the case that the Hamiltonian involves higher order terms. For such example, let us consider the Hamiltonian:

$$
\begin{aligned}
\widehat{H}_{C}= & \hbar \omega\left(\widehat{a}_{1}^{\dagger} \widehat{a}_{1}+\frac{1}{2}\right)+\hbar \Omega\left(\widehat{a}_{2}^{\dagger} \widehat{a}_{2}+\frac{1}{2}\right) \\
& +\hbar^{3 / 2} g(t) \hat{a}_{2}^{\dagger} \widehat{a}_{2}\left(\widehat{a}_{1}+\widehat{a}_{1}^{\dagger}\right) .
\end{aligned}
$$

The last term in this equation represents a nonlinear interaction which typically appears in a mechanical resonator coupled on superconducting stripline resonator [23]. In this case, it is difficult to know the adiabatic invariant due to the nonlinear term, and hence, to our knowledge, the use of perturbation theory is the best policy for studying quantum mechanical properties of this system. For a more detailed illustration for this, let us rewrite (23) as

$$
\widehat{H}_{C}=\widehat{H}_{A}^{\prime}+g(t) \sqrt{\frac{L_{n} \omega}{2}}\left(L_{s} \Omega \widehat{q}_{2}^{2} \widehat{q}_{1}+\frac{1}{L_{s} \Omega} \widehat{p}_{2}^{2} \widehat{q}_{1}\right),
$$

where

$$
\begin{aligned}
\widehat{H}_{A}^{\prime}= & \frac{\widehat{p}_{1}^{2}}{2 L_{n}}+\frac{\widehat{p}_{2}^{2}}{2 L_{s}}+\frac{1}{2} L_{n} \omega^{2} \widehat{q}_{1}^{2}+\frac{1}{2} L_{s} \Omega^{2} \widehat{q}_{2}^{2} \\
& -\hbar g(t) \sqrt{\frac{L_{n} \omega}{2}} \widehat{q}_{1} .
\end{aligned}
$$

By applying the method of previous development given for a simple superconducting resonator cavity, we can easily derive the adiabatic invariant of $\widehat{H}_{A}^{\prime}$. If it is possible to derive quantum solutions of the system described by $\widehat{H}_{A}^{\prime}$, we can use perturbation theory in order to derive the quantum solutions of $\widehat{H}_{C}$ after regarding the last term in (24) as the perturbation term [27].

The accuracy of conservation for invariant quantities is crucial for securing the validity of the associated analyses of classical and quantum mechanical characteristics of the system fulfilled on the basis of such invariants [16]. In this research, $\widehat{I}_{A}$ given in (6) is an exact invariant while $\widehat{I}_{B}$ given in (21) is an adiabatic invariant valid under the condition that $\lambda(t)$ is a slowly varying function. Both invariants are useful for investigating the classical and the quantum properties of each respective system. As a further task in the future, it may be a good subject to investigate quantum mechanical properties of superconducting qubit-oscillator systems employing the theory of invariants developed in this work.

\section{Conflict of Interests}

The author declares that there is no conflict of interests regarding the publication of this paper.

\section{Acknowledgment}

This research was supported by the Basic Science Research Program of the year 2015 through the National Research Foundation of Korea (NRF) funded by the Ministry of Education (Grant no. NRF-2013R1A1A2062907).

\section{References}

[1] V. V. Dodonov, V. I. Man'ko, and O. V. Man'ko, "Correlated states in quantum electronics (resonant circuit)," Journal of Soviet Laser Research, vol. 10, no. 5, pp. 413-420, 1989.

[2] T. Fujii, S. Matsuo, N. Hatakenaka, S. Kurihara, and A. Zeilinger, "Quantum circuit analog of the dynamical Casimir effect," Physical Review B: Condensed Matter and Materials Physics, vol. 84, no. 17, Article ID 174521, 2011.

[3] D. Zueco, G. M. Reuther, S. Kohler, and P. Hänggi, "Qubitoscillator dynamics in the dispersive regime: analytical theory beyond the rotating-wave approximation," Physical Review A: Atomic, Molecular, and Optical Physics, vol. 80, no. 3, Article ID 033846, 2009.

[4] M. Leib, F. Deppe, A. Marx, R. Gross, and M. J. Hartmann, "Networks of nonlinear superconducting transmission line resonators," New Journal of Physics, vol. 14, no. 7, Article ID 075024, 2012.

[5] S. He, Y.-Y. Zhang, Q.-H. Chen, X.-Z. Ren, T. Liu, and K.L. Wang, "Unified analytical treatments of qubit-oscillator systems," Chinese Physics B, vol. 22, no. 6, Article ID 064205, 2013.

[6] J. Q. You and F. Nori, "Atomic physics and quantum optics using superconducting circuits," Nature, vol. 474, no. 7353, pp. 589597, 2011

[7] I. Chiorescu, P. Bertet, K. Semba, Y. Nakamura, C. J. P. M. Harmans, and J. E. Mooij, "Coherent dynamics of a flux qubit coupled to a harmonic oscillator," Nature, vol. 431, no. 7005, pp. 159-162, 2004.

[8] A. Wallraff, D. I. Schuster, A. Blais et al., "Strong coupling of a single photon to a superconducting qubit using circuit quantum electrodynamics," Nature, vol. 431, no. 7005, pp. 162-167, 2004.

[9] S. M. Girvin, M. H. Devoret, and R. J. Schoelkopf, "Circuit QED and engineering charge-based superconducting qubits," Physica Scripta, vol. 2009, no. T137, Article ID 014012, 2009.

[10] S.-L. Zhu and Z. D. Wang, "Unconventional geometric quantum computation," Physical Review Letters, vol. 91, no. 18, Article ID 187902, 2003. 
[11] J. M. Burgers, "Die adiabatischen Invarianten bedingt periodischer Systeme," Annalen der Physik, vol. 357, no. 2, pp. 195-202, 1917.

[12] M. Kruskal, "Asymptotic theory of Hamiltonian and other systems with all solutions nearly periodic," Journal of Mathematical Physics, vol. 3, no. 4, pp. 806-828, 1962.

[13] H. R. Lewis Jr., "Class of exact invariants for classical and quantum time-dependent harmonic oscillators," Journal of Mathematical Physics, vol. 9, no. 11, pp. 1976-1986, 1968.

[14] D. A. Trifonov and A. K. Angelow, "Dynamical invariants and Robertson-Schrödinger correlated states of electromagnetic field in nonstationary linear media," AIP Conference Proceedings, vol. 1340, pp. 221-233, 2011.

[15] A. M. Dykhne, "Quantum transitions in the adiabatic approximation," Soviet Physics-JETP, vol. 11, pp. 411-415, 1960.

[16] A. I. Neishtadt, "On the accuracy of conservation of the adiabatic invariant," Journal of Applied Mathematics and Mechanics, vol. 45, no. 1, pp. 58-63, 1981.

[17] T. Su, "On the accuracy of conservation of adiabatic invariants in slow-fast Hamiltonian systems," Regular and Chaotic Dynamics, vol. 17, no. 1, pp. 54-62, 2012.

[18] M. Nambu, S. V. Vladimirov, and H. Schamel, "On the physics of the plasma maser," Physics Letters A, vol. 178, no. 5-6, pp. 400406, 1993.

[19] C. Zhang, Z. Wei, and A. Papageorgiou, "Adiabatic quantum counting by geometric phase estimation," Quantum Information Processing, vol. 9, no. 3, pp. 369-383, 2010.

[20] J. D. Biamonte, V. Bergholm, J. D. Whitfield, J. Fitzsimons, and A. Aspuru-Guzik, "Adiabatic quantum simulators," AIP Advances, vol. 1, no. 2, Article ID 022126, 2011.

[21] R. Chakrabarti and B. V. Jenisha, "Quasi-Bell states in a strongly coupled qubit-oscillator system and their delocalization in the phase space," Physica A, vol. 435, pp. 95-110, 2015.

[22] B. D. Vujanovic and S. E. Jones, Variational Methods in Nonconservative Phenomena, Academic Press, New York, NY, USA, 1989.

[23] K. Jacobs, H. I. Nurdin, F. W. Strauch, and M. James, "Frequency conversion: side-band cooling, state-swapping, and coherent control of mechanical resonators," http://arxiv.org/abs/ 1003.2653.

[24] B. Peropadre, D. Zueco, F. Wulschner et al., "Tunable coupling engineering between superconducting resonators: from sidebands to effective gauge fields," Physical Review B: Condensed Matter and Materials Physics, vol. 87, no. 13, Article ID 134504, 12 pages, 2013.

[25] E. Gozzi, "Classical and quantum adiabatic invariants," Physics Letters B, vol. 165, no. 4-6, pp. 351-354, 1985.

[26] I. A. Malkin, V. I. Man'Ko, and D. A. Trifonov, "Linear adiabatic invariants and coherent states," Journal of Mathematical Physics, vol. 14, no. 5, pp. 576-582, 1973.

[27] J. R. Choi and Y. Choi, "Stochastic quantization of Brownian particle motion obeying Kramers equation," Journal of the Physical Society of Japan, vol. 79, no. 6, Article ID 064004, 2010. 


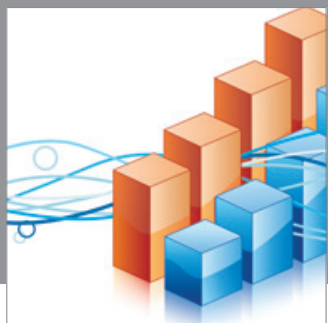

Advances in

Operations Research

mansans

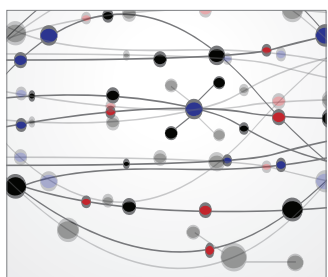

The Scientific World Journal
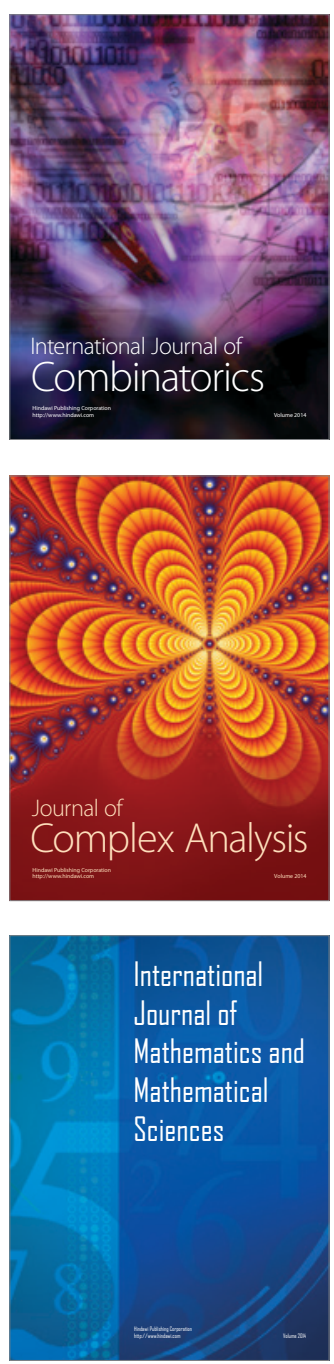
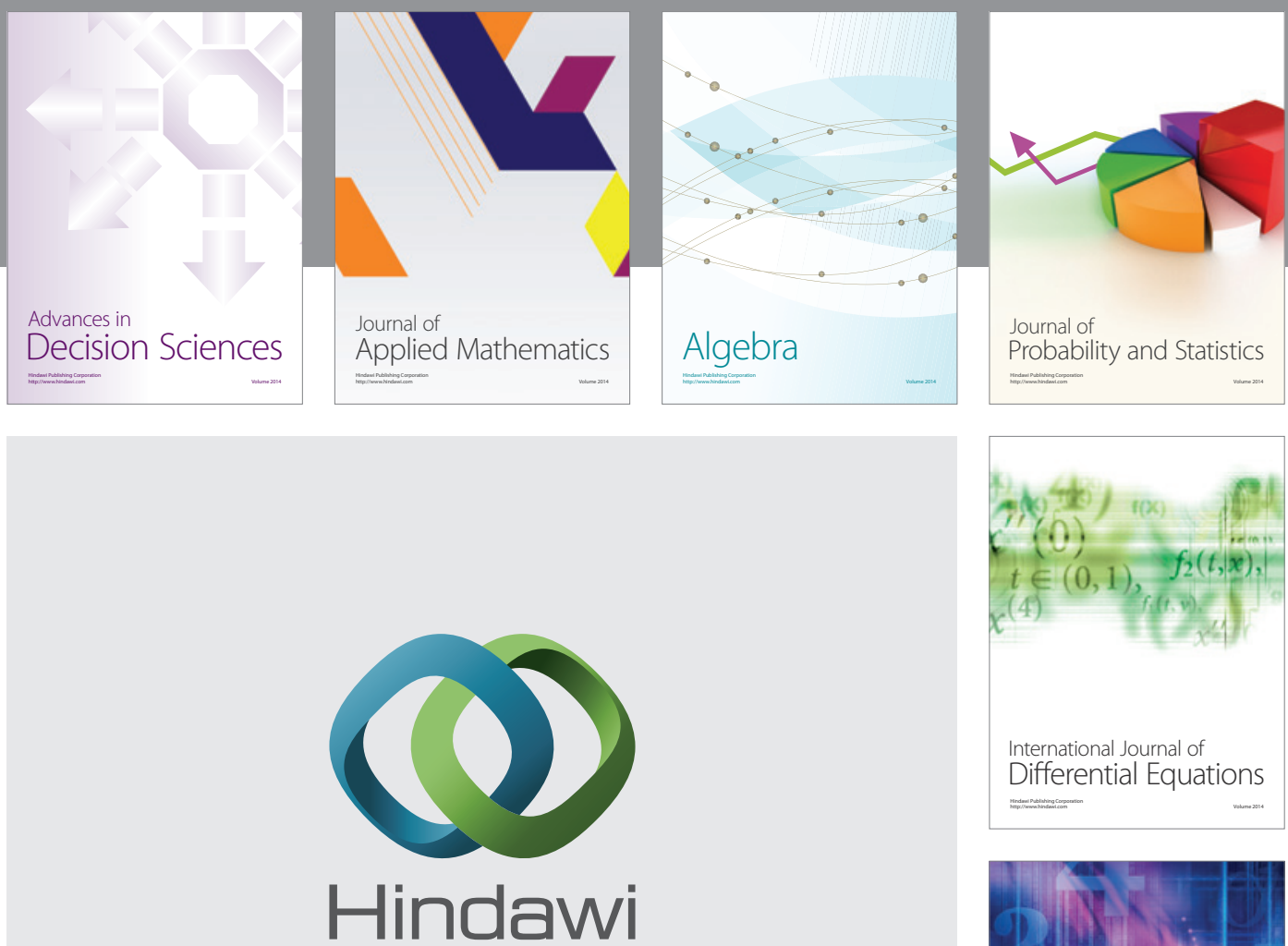

Submit your manuscripts at http://www.hindawi.com
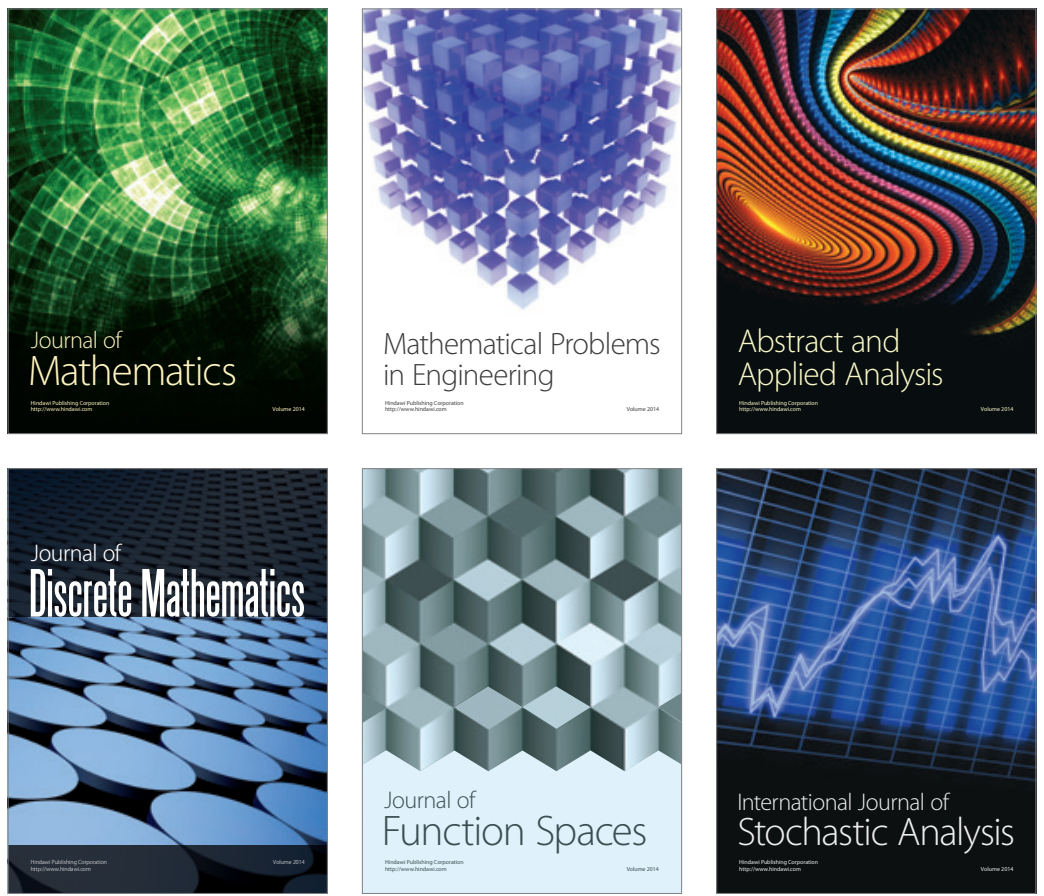

Journal of

Function Spaces

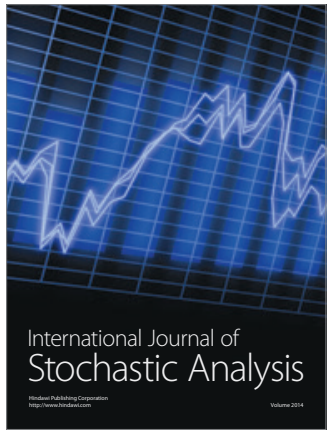

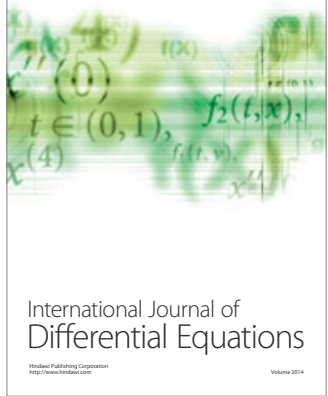
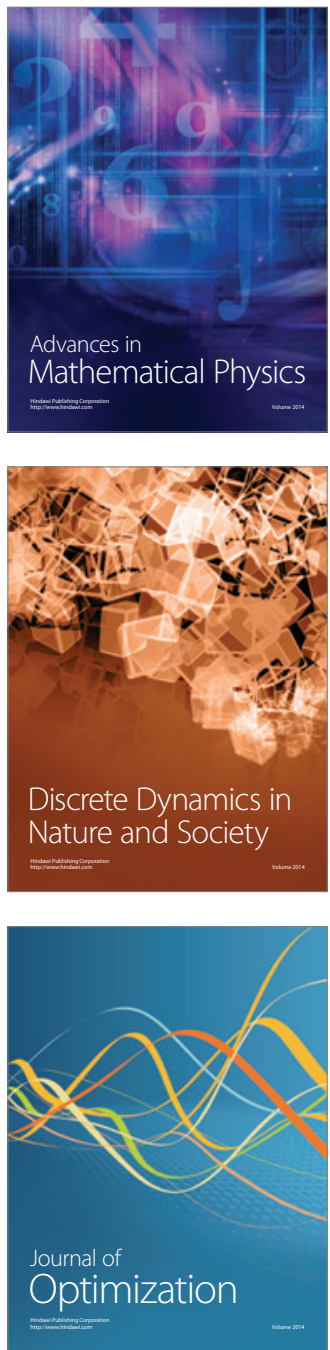